INPLASY

PROTOCOL

To cite: Wang et al. Efficacy and safety of acupuncture combined with Chinese herbal medicine traditional chinese medicine for post-stroke cognitive impairment: a protocol for a systematic review and meta-analysis. Inplasy protocol 202220062. doi: 10.37766/inplasy2022.2.0062

Received: 16 February 2022

Published: 16 February 2022

Corresponding author: Ying Wang

ml89688466@163.com

Author Affiliation:

Chengdu University of TCM.

Support: 030058004.

Review Stage at time of this submission: Preliminary searches.

Conflicts of interest: None declared.

\section{Efficacy and safety of acupuncture combined with Chinese herbal medicine traditional chinese medicine for post- stroke cognitive impairment: a protocol for a systematic review and meta-analysis}

\author{
Wang, Y1; Guo, S2; Xiao, ML3; Zhang, $\mathrm{H}^{4}$.
}

Review question / Objective: The purpose of this study is to assess the effectiveness and safety of the acupuncture combined with TCM in the treatment of post-stroke cognitive impairment.All randomized controlled trials eligible for acupuncture combined with traditional chinese medicine for post-stroke cognitive impairment were included in this study. Eligibility criteria: Western diagnostic criteria for cognitive impairment after stroke:The diagnostic criteria of cerebral apoplexy refer to the diagnostic criteria of cerebral apoplexy in "Main Points of Diagnosis of Various Cerebrovascular Diseases" revised by the Chinese Medical Association in 1995 at the fourth National Academic Conference on cerebrovascular diseases. It also conforms to the diagnostic criteria of "Expert Consensus on the Prevention and treatment of Post-stroke Cognitive Impairment in China" published by Chinese Stroke Journal in 2020.

INPLASY registration number: This protocol was registered with the International Platform of Registered Systematic Review and Meta-Analysis Protocols (INPLASY) on 16 February 2022 and was last updated on 16 February 2022 (registration number INPLASY202220062).

\section{INTRODUCTION}

Review question / Objective: The purpose of this study is to assess the effectiveness and safety of the acupuncture combined with TCM in the treatment of post-stroke cognitive impairment. All randomized controlled trials eligible for acupuncture combined with traditional chinese medicine for post-stroke cognitive impairment were included in this study.

Condition being studied: Study selection, data extraction and quality assessment will be performed by 2 reviewers independently. Bias risk assessment and data synthesis 
will be performed using the Review Manager software (RevMan, version 5.3.5) and $\mathbf{R}$ (version 3.6.1) software.

\section{METHODS}

Participant or population: Patients with post-stroke cognitive impairment.

Intervention: Acupuncture combined with Chinese herbal medicine traditional chinese medicine.

Comparator: Control group was treated with conventional western medicine treatment plan.

Study designs to be included: Randomized controlled trial.

Eligibility criteria: Western diagnostic criteria for cognitive impairment after stroke:The diagnostic criteria of cerebral apoplexy refer to the diagnostic criteria of cerebral apoplexy in "Main Points of Diagnosis of Various Cerebrovascular Diseases" revised by the Chinese Medical Association in 1995 at the fourth National Academic Conference on cerebrovascular diseases. It also conforms to the diagnostic criteria of "Expert Consensus on the Prevention and treatment of Post-stroke Cognitive Impairment in China" published by Chinese Stroke Journal in 2020.

Information sources: The Cochrane Library, PubMed, Embase, Medline, Web of Science, China National Knowledge Infrastructure (CNKI), China Biology Medicine (CBM), Wan Fang data and the Chinese Science and Technology Periodical Database (VIP).

Main outcome(s): The primary outcome of the study is that cognitive function will be assessed by the Montreal Cognitive Assessment scale (MoCA), and the MiniMental State Examination (MMSE).

Additional outcome(s): The secondary outcomes will include the following: 1.Attentional performance: (Test of Attentional Performance,TAP); 2.Memory: (Wechsler Memory Scale, WMS); 3.Quality of life: Short Form Survey Instrument (SF-36), Fugl-Meyer Motor Assessment, Activities of daily living (ADL); 4.Adverse events related to acupuncture combined with traditional Chinese medicine treatment (e.g., stuck needle, bleeding, dizziness).

Quality assessment / Risk of bias analysis: The Cochrane Collaboration Tool.

Strategy of data synthesis: Assessment of heterogeneity: We will use 12 value of the Chi-Squared test to detect the heterogeneity of included studies. If $12 \leq 50 \%$, it means acceptable homogeneity. Otherwise, if $12>50 \%$, it means significant heterogeneity. Data synthesis: All data synthesis and analysis will use Review Manager 5.3 (RevMan), We will use a fixedeffects model if there is no statistical heterogeneity between the results, and perform meta-analysis. We will use a random-effects model if there is statistical heterogeneity between the results. If there is significant heterogeneity, we will use subgroup analysis or sensitivity analysis.

Subgroup analysis: Type of acupuncture stimulation, different TCM decoctions, subject (age, gender), and duration of treatment will be considered.

Sensitivity analysis: Review Manager5.3 was used for sensitivity analysis of the data, and each article was excluded and analyzed one by one to observe the change of heterogeneity.

Country(ies) involved: China.

Keywords: post-stroke cognitive impairment, acupuncture, traditional chinese medicine.

Contributions of each author:

Author 1 - Ying Wang.

Author 2 - Sha Guo.

Author 3 - Meng-lu Xiao.

Author 4 - Hong Zhang. 